\title{
The Centre of Gravity of a Circular Arc.
}

By Mr G. E. Crawford, M.A.

To find the Centre of Gravity of a Ciroular Arc.

Let $a$ (Fig. 12) be the radius, $2 a$ the angle at the centre, AB the arc, of total mass $m, G$ its centre of gravity symmetrically situated.

Imagine the arc to be part of a circle of string rotating uniformly with velocity $u$ round $\mathrm{C}$ and of linear density $\rho$

Then if $T$ be the Tension at either extremity

Resolving $2 T \sin a=$ Force to centre

$$
\begin{aligned}
& =m d w^{2}=2 \rho a \alpha d w^{2} \\
\therefore \mathrm{T} & =a \rho w^{2} \frac{\alpha d}{\sin \alpha}
\end{aligned}
$$

But $\mathrm{T}$ being constant, this formula must be constant, and $\therefore$ true for all values of $\alpha$

$$
\therefore \frac{a d}{\sin \alpha} \text { is constant. }
$$

But when $\alpha=0$ its value is $a$

$$
\therefore \quad d=a \frac{\sin \alpha}{a} \text {. }
$$

\section{A Demonstration of the Apparatus used in Practical Skiagraphy by the Röntgen Rays.}

By Dr Harry Rainy. 